\title{
In and out of the rRNA genes: characterization of Pokey elements in the sequenced Daphnia genome
}

Tyler A Elliott ${ }^{1 *+}$, Deborah E Stage ${ }^{2,3+}$, Teresa J Crease ${ }^{1}$ and Thomas H Eickbush ${ }^{2}$

\begin{abstract}
Background: Only a few transposable elements are known to exhibit site-specific insertion patterns, including the well-studied R-element retrotransposons that insert into specific sites within the multigene rDNA. The only known rDNA-specific DNA transposon, Pokey (superfamily: piggyBac) is found in the freshwater microcrustacean, Daphnia pulex. Here, we present a genome-wide analysis of Pokey based on the recently completed whole genome sequencing project for $D$. pulex.
\end{abstract}

Results: Phylogenetic analysis of Pokey elements recovered from the genome sequence revealed the presence of four lineages corresponding to two divergent autonomous families and two related lineages of non-autonomous miniature inverted repeat transposable elements (MITEs). The MITEs are also found at the same 28S rRNA gene insertion site as the Pokey elements, and appear to have arisen as deletion derivatives of autonomous elements. Several copies of the full-length Pokey elements may be capable of producing an active transposase. Surprisingly, both families of Pokey possess a series of 200 bp repeats upstream of the transposase that is derived from the rDNA intergenic spacer (IGS). The IGS sequences within the Pokey elements appear to be evolving in concert with the rDNA units. Finally, analysis of the insertion sites of Pokey elements outside of rDNA showed a target preference for sites similar to the specific sequence that is targeted within rDNA.

Conclusions: Based on the target site preference of Pokey elements and the concerted evolution of a segment of the element with the rDNA unit, we propose an evolutionary path by which the ancestors of Pokey elements have invaded the rDNA niche. We discuss how specificity for the rDNA unit may have evolved and how this specificity has played a role in the long-term survival of these elements in the subgenus Daphnia.

Keywords: Transposons, Daphnia, Pokey, Ribosomal DNA, Insertion specificity

\section{Background}

Transposable elements (TEs) are found in nearly all organisms and often comprise substantial portions of eukaryotic genomes [1]. Many TEs insert into locations throughout the genome, while others insert preferentially into specific sequences. A site preferred by nonlong terminal repeat (non-LTR) retrotransposons is the locus encoding rRNA [2]. Pokey is the only example of a DNA transposon known to insert specifically in rDNA. Pokey inserts into the same $28 \mathrm{~S}$ gene region that is

\footnotetext{
*Correspondence: telliott@uoguelph.ca

${ }^{\dagger}$ Equal contributors

'Department of Integrative Biology, University of Guelph, Guelph, ON N1G 2W1, Canada

Full list of author information is available at the end of the article
}

highly targeted by non-LTR elements [3]. Insertion of any of these elements is expected to disrupt the production of functional rRNA from the inserted units.

rDNA is comprised of hundreds to thousands of units arrayed in tandem encoding one copy each of the core $18 \mathrm{~S}, 5.8 \mathrm{~S}$ and $28 \mathrm{~S}$ rRNAs. The many copies of each rRNA gene show high sequence identity, the product of recombinational processes termed concerted evolution (reviewed in [2]). The primary mechanism conferring high identity between copies is unequal crossing over, which also generates the large variation in rDNA copy number observed between members of the same species [4]. The combined processes of concerted evolution and selection against inserted units require that any element with a long-term presence in the rDNA unit regularly 
generate new insertions to avoid being eliminated from the locus $[4,5]$.

Pokey elements are members of the piggyBac superfamily of DNA-mediated TEs that insert into TTAA target sequences $[3,6]$. This element was first identified in the cladoceran crustacean Daphnia pulex, and is now known to be widespread throughout the subgenus Daphnia. Unlike most DNA TEs, Pokey elements have undergone stable vertical inheritance for millions of years [7]. To the best of our knowledge, the only other organisms in which open reading frames (ORFs) similar to those in Pokey have been found are the silkmoth Bombyx mori [8], the tunicate Ciona savignyi [9], and the rotifer Adineta vaga [10]. Pokey elements have been found at multiple TTAA insertion sites throughout the genome and thus, like other piggyBac elements, appear to require little additional conservation of target sites $[3,11]$. Nevertheless, Pokey have been repeatedly found at just one location in the $28 \mathrm{~S}$ genes despite the presence of over 30 TTAA motifs in the entire rDNA unit. While this finding might suggest that properties in addition to TTAA are preferred for Pokey insertion, the frequency of independent Pokey insertions in the rDNA locus is not known. Thus, it is unclear whether rDNA acts as a sink or source for Pokey elements, or whether there is free and on-going exchange between Pokey elements in and outside the rRNA genes.
In this study, we used the original sequencing reads available from the Daphnia genome sequencing project, available at the Trace Archives at GenBank, as well as the annotated scaffold sequences to study Pokey elements and their interactions with $28 \mathrm{~S}$ genes. The Pokey elements are divided into two divergent lineages each possessing a unique inverted terminal repeat (ITR) structure. Both lineages carry repeated copies of a segment from the intergenic spacer (IGS) region of the rDNA unit. In addition, two lineages of non-autonomous miniature inverted repeat transposable elements (MITEs) are present at the Pokey site in $28 \mathrm{~S}$ genes, and elsewhere in the genome. Finally, weak target sequence preferences for Pokey and the MITEs were found that are consistent with the site that is targeted in the $28 \mathrm{~S}$ gene. We suggest that Pokey elements have evolved specificity for their $28 \mathrm{~S}$ gene insertion site and their presence at this site has played a key role in their long term survival in Daphnia by acting as a source for Pokey and their MITEs throughout the genome.

\section{Results}

\section{rDNA sequence variation}

Assembly of a consensus rDNA repeating unit from the Daphnia genome revealed a gene organization typical of most eukaryotes (Figure 1A, Additional file 1). The IGS separating transcription units in Daphnia starts with an $840 \mathrm{bp}$ non-repetitive region, followed by a series of 323

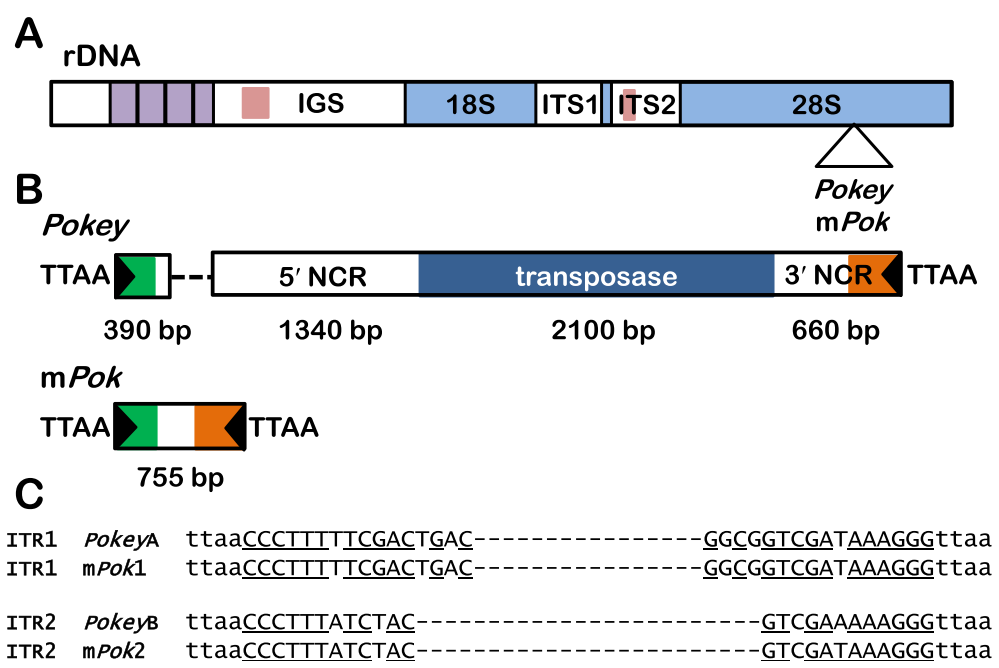

Figure 1 The structure of rDNA and its transposons in the Daphnia genome sequence. (A) The rDNA is an array of transcription units repeated in tandem and separated by the intergenic spacer (IGS), which contains a repeated sequence (purple boxes), as well as non-repeated regions. Pink boxes in the IGS and internal transcribed spacer (ITS)2 indicate the position of sequences found in the $5^{\prime}$ noncoding region (NCR) of Pokey elements. (B) The structure of Pokey and Pokey-derived miniature inverted repeat transposable elements (mPok), which insert in a specific TTAA site in $28 \mathrm{~S}$ rRNA genes. The approximate length of each region of a canonical autonomous Pokey element is given below the diagram. The black triangles at each end represent the inverted terminal repeats (ITRs). The dashed line in Pokey represents the highly length-variable region composed of both repetitive and unique sequences (more detail is provided in Figure 5). The green and orange regions correspond to regions that are similar in Pokey and mPok, the sequences of which are shown in Figure 2. (C) Sequence of the two types of ITRs. Complementary regions between the imperfect $5^{\prime}$ and $3^{\prime}$ ITRs are underlined. The TTAA target site duplication is shown in lower case. 
bp repeats, and ends in a non-repetitive 3,115 bp region. The last region should include an external transcribed spacer, but the transcription start site is not known. Ambrose and Crease [12] have shown that the 323 bp repeats are composed of two subrepeats of $200 \mathrm{bp}$ and 123 bp, which usually (but not always) alternate with each other. Most (58\%) IGSs in the genome sequence contain three 123-bp and four 200-bp repeats with the remaining IGSs containing more copies of each repeat.

Concerted evolution is expected to maintain very high sequence identity among all copies of the rDNA unit. The rDNA transcription units (external transcribed spacer through the $28 \mathrm{~S}$ gene) in sequenced genomes of Drosophila [13] and Nasonia [14] contain from 3 to 18 sites in which sequence variants are present in over $3 \%$ of units. In contrast, the Daphnia rDNA transcription unit and a $500 \mathrm{bp}$ non-repetitive region from the IGS contain no sequence variants at the $3 \%$ threshold. This especially low level of rDNA variation is consistent with the very high level of homozygosity at allozyme and microsatellite markers observed in the sequenced Daphnia isolate [15], the low level of sequence variation in $28 \mathrm{~S}$ genes from D. pulex in natural populations [16], and the high rate of recombination observed in the rDNA of a closely related species, Daphnia obtusa [17].

\section{Pokey elements in $28 \mathrm{~S}$ genes and the genome}

A consensus sequence for Pokey copies was assembled from the original sequence reads of the $D$. pulex genome (Additional file 1). We also identified 69 elements containing intact ITRs at both ends from the annotated genome scaffolds at wFleabase. We aligned these sequences to two copies of Pokey elements from $D$. pulicaria rDNA, which were designated pcPokeyS $(5 \mathrm{~kb})$ and $\mathrm{pcPokey \textrm {L }}(6.6 \mathrm{~kb})$ [3]. As shown in Figure 1B, the Pokey elements contain either 12 or 16 bp imperfect ITRs, a 5 ' non-coding region (NCR), an ORF encoding a putative transposase, and a $3^{\prime}$ NCR. The $D$. pulex copies were up to $9,800 \mathrm{bp}$ in length (Additional file 2) with the majority of the length variation occurring in the $5^{\prime} \mathrm{NCR}$ (discussed below). Excluding this repetitive region, the canonical Pokey element is approximately 4,500 bp.

We also identified an additional 91 incomplete sequences from 400 to $4,400 \mathrm{bp}$ in length that lack either the $5^{\prime}$ or $3^{\prime}$ ITR, or both. The total number of Pokey elements based on these genomic searches was 160, similar to the 175 estimated by comparing the depth of coverage of Pokey sequence reads to the average coverage of single copy genes [15]. We estimate that six of the 175 copies are inserted into $28 \mathrm{~S}$ genes and they all have the 12-bp ITRs.

A second type of sequence was also found at the Pokey insertion site in $28 \mathrm{~S}$ genes and elsewhere in the genome. These elements were approximately $750 \mathrm{bp}$ in length and contained sequences corresponding to the ends of the Pokey elements (Figure 1B). These shorter elements could be divided into two groups that contain the same imperfect 12 or 16 bp ITRs found in full-length Pokey elements, and thus are designated as MITEs. Sequence identity between the Pokey and MITEs extends for 160 bp at their $5^{\prime}$ ends and $350 \mathrm{bp}$ at their $3^{\prime}$ ends (Figure 2). These regions contain repeat sequences that have been found in other piggyBac elements [18]. The central 250 bp region of the MITEs has no readily observed similarity to that of the Pokey elements.

Like the Pokey elements, MITEs found outside $28 \mathrm{~S}$ genes also target TTAA sites, suggesting that they use the transposase of Pokey elements. We hereafter refer to these MITEs as mPok. About 25 to 30 copies of these $\mathrm{m}$ Pok were found in $28 \mathrm{~S}$ genes, all with $12-\mathrm{bp}$ ITRs. The total genome contains 90 to 110 copies with $60 \mathrm{mPok}$ sequences in the assembled scaffolds (Additional file 2).

\section{Cluster analysis of Pokey and mPok elements}

A Neighbor-joining (NJ) tree was constructed from the consensus rDNA Pokey sequence, the pcPokeyS and L sequences from $D$. pulicaria and 29 Pokey elements from the assembled genome scaffolds of $D$. pulex that contained full-length transposase sequences and less than $5 \%$ ambiguous base-calls. The length-variable region of the $5^{\prime}$ NCR (Figure 1B) was omitted from this analysis. The tree revealed two clusters with high bootstrap support, which will be referred to as the PokeyA and B families (Figure 3). The PokeyA cluster contains the two pcPokey elements described from $D$. pulicaria [3]. The Pokey $\mathrm{B}$ cluster contains a second paralogous lineage of Pokey elements previously identified by Penton and Crease [7] from D. obtusa (Additional file 3). All PokeyA elements contain the 16-bp ITR1, while PokeyB elements have the 12-bp ITR2. Average sequence divergence between the 11 Pokey A elements is 5.9\% while average divergence between the 18 PokeyB elements is $5.0 \%$ (Table 1). Divergence between the two groups averages $39.9 \%$. Based on the sequence of their ITRs, $11(15.9 \%)$ of the 69 elements obtained from the annotated scaffolds are PokeyA while the remaining 58 (84.1\%) are PokeyB (Additional file 2).

An NJ tree was also constructed with all $60 \mathrm{mPok}$ sequences identified in the assembled scaffolds and the consensus rDNA mPok sequence. Two clusters with high bootstrap support were again observed (Figure 4), one sharing the 16 bp ITR1 with PokeyA (designated $\mathrm{mPok} 1$ ) and the other sharing the 12 bp ITR2 with PokeyB (designated $\mathrm{mPok} 2$ ). mPok2 elements (46 copies) are over three times as numerous as mPok1 elements (14 copies). Intragroup sequence divergence for $\mathrm{mPok} 1$ is only $2.2 \%$. In the case of $\mathrm{mPok} 2$, there is a large cluster of elements (mPok2a, Figure 4) with low average 


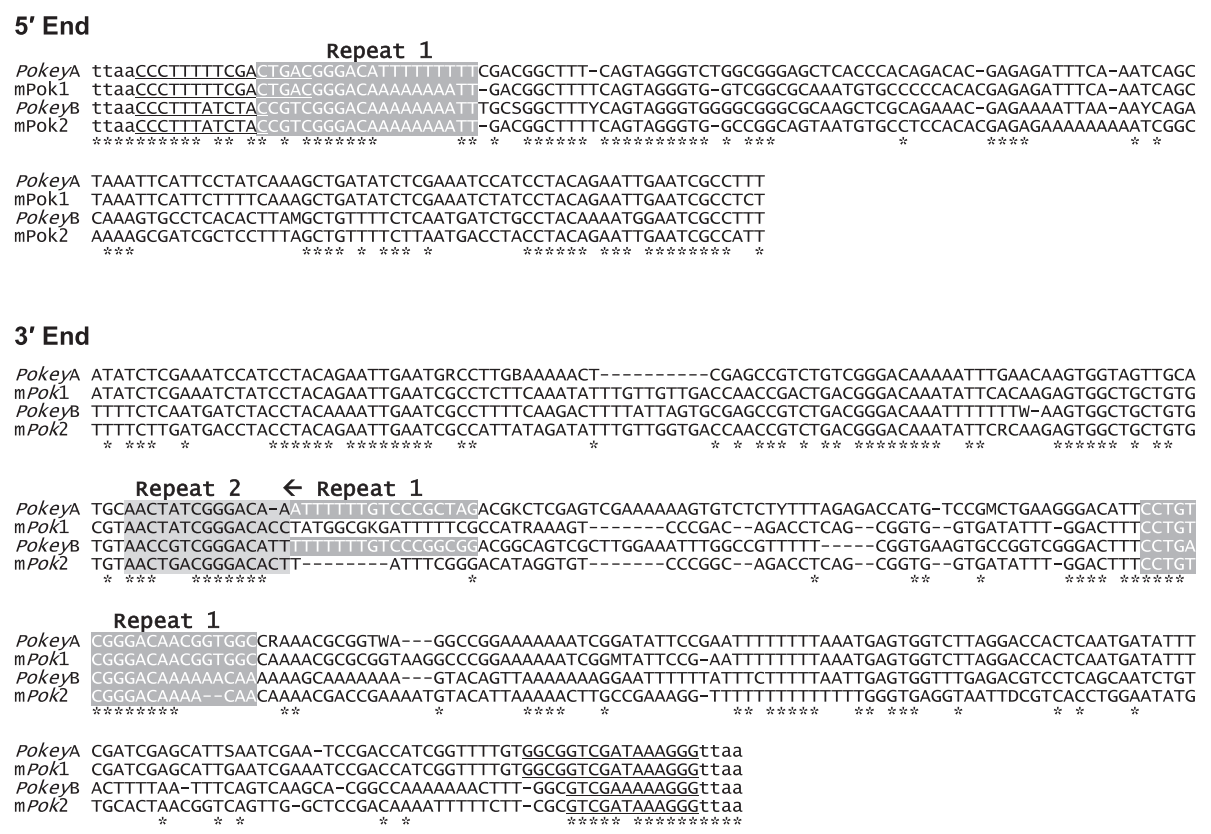

Figure 2 Partial alignment of Pokey and mPok consensus sequences. Nucleotides conserved across all four sequences are marked with asterisks. The inverted terminal repeats are underlined. Repeat 1 and 2 refer to sequences shown in Additional file $6 . B=C, G$ or $T, M=A$ or $C$, $\mathrm{R}=\mathrm{A}$ or $\mathrm{G}, \mathrm{W}=\mathrm{A}$ or $\mathrm{T}$. mPok, Pokey-derived miniature inverted repeat transposable element.

sequence divergence (3.2\%) and a second group (mPok2b) with much higher divergence (20.4\%, Table 1). Inspection of the $\mathrm{mPok} 2 \mathrm{~b}$ sequences reveals few intact ITRs and numerous insertions and deletions suggesting that they represent older copies of $\mathrm{mPok} 2$ that are no longer able to transpose. Divergence between $\mathrm{mPok} 1$ and $\mathrm{mPok} 2 \mathrm{a}$ is 24.9\% (Table 1), somewhat lower than the divergence estimates between full length PokeyA and PokeyB elements.

\section{Characterization of the Pokey transposase}

The ORF from the pcPokeyL element was originally reported by Penton and colleagues [3] to be 1,461 bp encoding a protein of 487 amino acids. However, this coding region was suggested to contain a $68 \mathrm{bp}$ intron ( $\mathrm{Y}$ Bigot, personal communication), which when spliced from an RNA transcript would enable the production of a 668 amino acid protein. Pokey elements from D. pulex also appear to have this intron, which ranged in size from 68 to $74 \mathrm{bp}$ in PokeyA and from 79 to $84 \mathrm{bp}$ in PokeyB. Analysis of Pokey RNA transcripts by RT-PCR confirmed that the putative intron sequence can be spliced out [19].

PokeyA and Pokey $\mathrm{B}$ transposase genes encode conserved motifs shared among the transposase genes of diverse piggyBac elements [20]. These include a DDD (aspartic acid) motif (amino acid residues 436, 544 and 659) that is considered essential for transposase activity, an imperfect zinc finger motif that is believed to be either a chromatin-interacting Plant Homeo Domain or a protein-protein interaction domain, and a putative nuclear localization signal. Keith and colleagues [20] identified a fourth D residue C-terminal to the catalytic DDD triad. When they mutated this charged $D$ to an uncharged $\mathrm{N}$ (asparagine) in a piggyBac construct, they observed a significant reduction in the transposition rate. This fourth residue is $\mathrm{N}$ instead of $\mathrm{D}$ in the $D$. pulicaria and D. pulex Pokey elements (Additional file 4). Partial sequences of Pokey transposase genes from other species in the subgenus Daphnia [7] all encode an N at this site.

Of the 69 elements identified from the assembled scaffolds, two PokeyA and two PokeyB elements were identified that may encode transposition-competent transposases (identified on the NJ tree in Figure 3). The ORF of these elements lacked premature stop codons and contained all features known or inferred to be important for the transposition of piggyBac.

\section{Repeated sequences in Pokey and mPok}

Penton and colleagues [3] noted the presence of a 200bp repeat sequence (A repeats) in the $5^{\prime} \mathrm{NCR}$ of $D$. pulicaria Pokey elements that was derived from the IGS region of the rDNA unit. We also observed A repeats in the Pokeys from D. pulex and note that they are usually preceded by a 48 bp sequence derived from ITS2 (Figure 5; see Figure 1 for the location of these sequences in the rDNA unit). The ITS2 repeat was termed $\mathrm{C}$ to differentiate it from an IGS-derived sequence previously designated as B in the pcPokeyL element [3]. All 


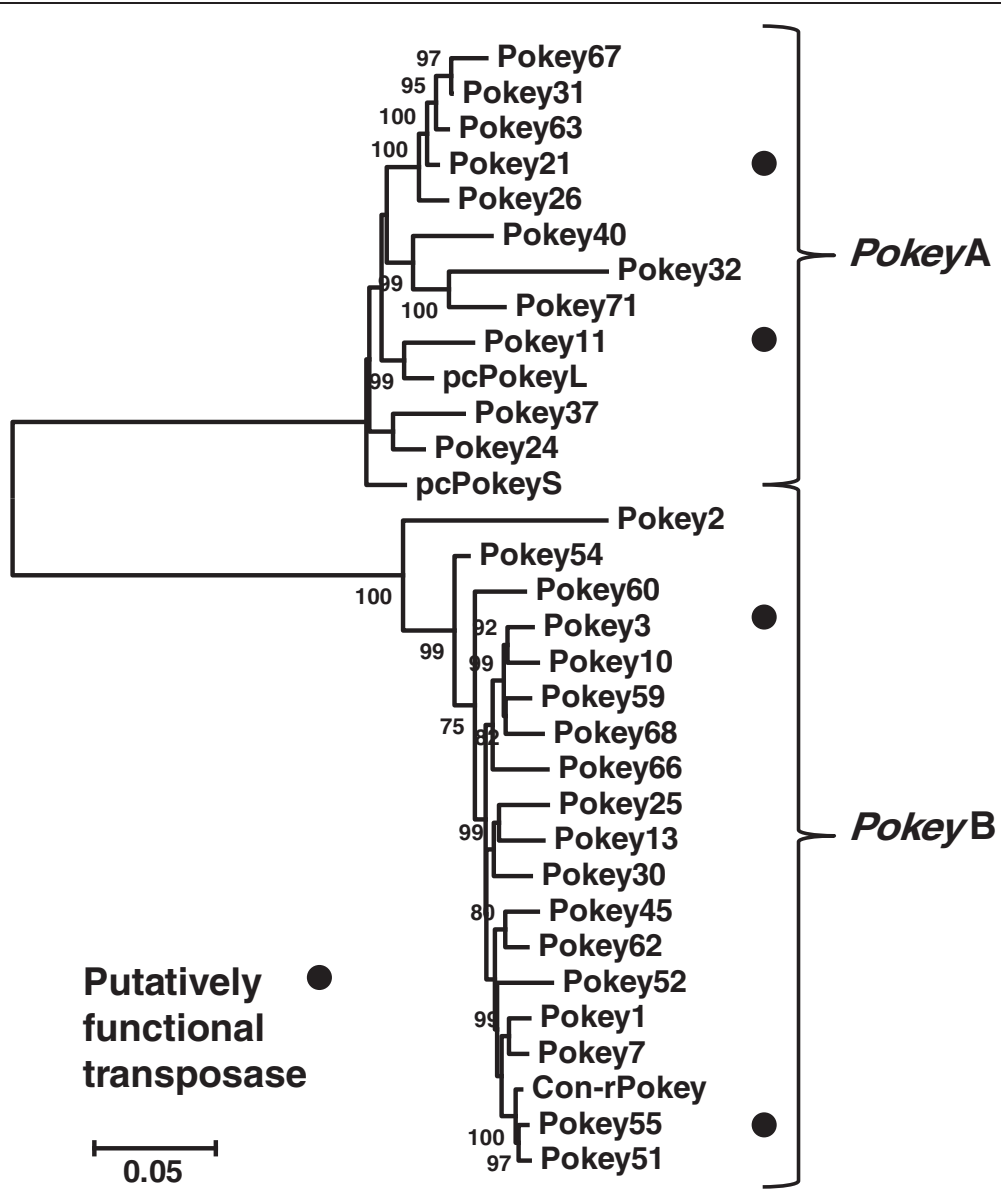

Figure 3 Unrooted Neighbor-joining tree of full-length Pokey elements from the Daphnia genome. The 27 elements containing putative transposase genes and less than 5\% ambiguous bases, and the consensus rDNA element (Con-rPokey) are included in the tree. The elements form two well-supported clusters that correspond to the inverted terminal repeat (ITR) 1 and ITR2 sequences (Figure 1C). All positions containing gaps and missing data were eliminated in pairwise comparisons. Bootstrap values greater than 70 are indicated at the nodes of the tree.

but one Pokey element from the annotated scaffolds contain both $\mathrm{A}$ and $\mathrm{C}$ repeats with their copy number varying between 2 and 5 per element. Due to the possibility of assembly errors in repeat regions, we cannot be certain of the exact repeat configuration of each element. However, the evidence does suggest these regions are highly variable among elements. In addition, large tracts

Table 1 Sequence divergence between Pokey elements from the Daphnia genome

\begin{tabular}{lccccc}
\hline Lineage & PokeyA & PokeyB & mPok1 & mPok2a & mPok2b \\
\hline PokeyA & 0.059 & 0.399 & & & \\
PokeyB & & 0.050 & & & \\
mPok1 & & & 0.032 & & \\
mPok2a & & & 0.249 & 0.022 & \\
mPok2b & & & 0.323 & 0.200 & 0.204
\end{tabular}

Estimates were calculated using the Kimura 2-parameter and pairwise deletion options in MEGA4. Estimates are based on separate alignments of PokeyA and PokeyB elements (28 sequences) and of mPok (61 sequences). mPok, Pokeyderived miniature inverted repeat transposable element. of additional sequences derived from areas of the Daphnia genome outside the rDNA units were inserted between the A repeats of several Pokey elements (Figure 5) suggesting that the $5^{\prime} \mathrm{NCR}$ frequently acquires nonelement sequences from the genome.

We aligned the A repeat region of three $D$. pulex and three D. pulicaria ribosomal IGS sequences [12] to A repeats from all available Pokey elements (Additional file 5) and generated an NJ tree (Figure 6). The IGS sequences do not cluster separately from the Pokey repeats, nor do repeats from PokeyA and PokeyB elements form separate clusters relative to one another. Mean sequence divergence among the A repeats from all Pokey elements is only $5.3 \%$ (range 0 to $23.9 \%$ ). In comparison, intraspecific sequence divergence in the region of the $D$. pulex and D. pulicaria IGS similar to A repeats is $1.8 \%$ [12]. This high sequence identity among the A repeats of the Pokey elements is in sharp contrast to the transposase sequences where mean nucleotide sequence divergence between the Pokey A and B families is nearly 


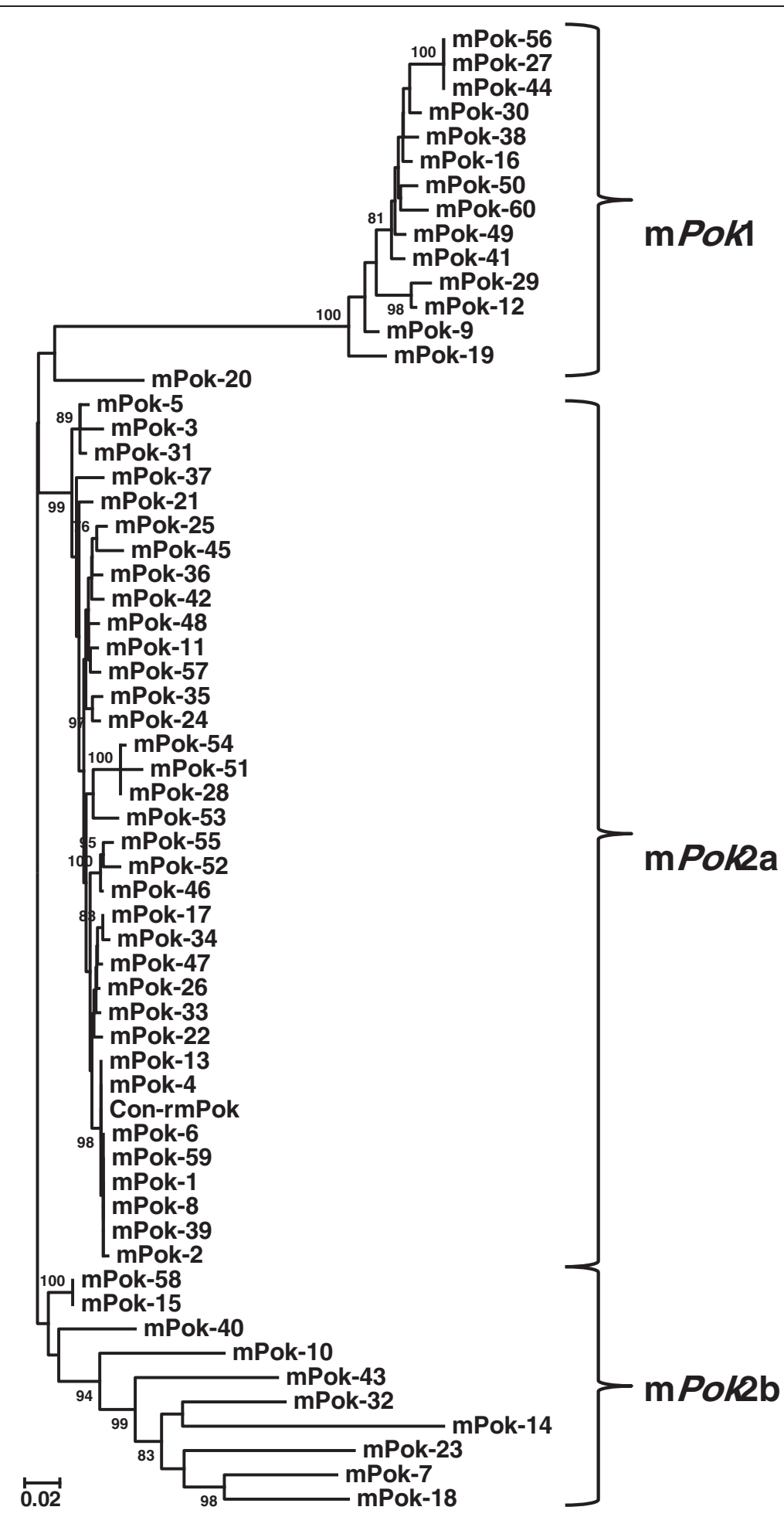

Figure 4 Unrooted Neighbor-joining tree of $61 \mathrm{mPok}$ sequences from the Daphnia genome. Con-rmPok is the consensus element in rDNA. The elements form two main clusters, denoted mPok1 and mPok2 that correspond to elements with inverted terminal repeat (ITR) 1 and ITR2 sequences, respectively (Figure 1C). All positions containing gaps and missing data were eliminated in pairwise comparisons. Bootstrap values greater than 70 are indicated at the nodes of the tree. mPok, Pokey-derived miniature inverted repeat transposable element. 


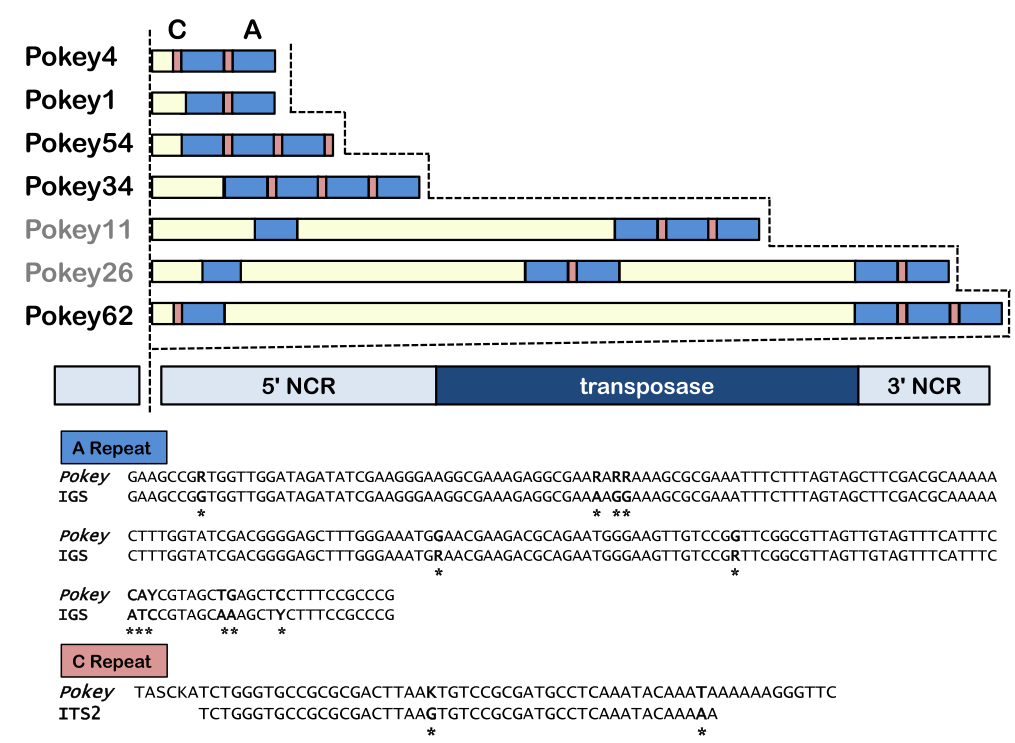

Figure 5 The $\mathbf{5}^{\prime}$ length-variable region of Pokey elements from the Daphnia genome. Examples of the $5^{\prime}$ length-variable region containing $\mathbf{A}$ and $\mathbf{C}$ repeats are drawn to scale above the canonical element, also drawn to scale. PokeyA element names are in grey, and PokeyB element names are in black. The $\mathbf{A}$ repeats (approximately 200 bp) are similar to a sequence in the Daphnia intergenic spacer (IGS) while $\mathbf{C}$ repeats (approximately $50 \mathrm{bp}$ ) are similar to a sequence in the Daphnia internal transcribed spacer (ITS)2. Asterisks and bold type indicate variable positions in the aligned sequences. NCR, non-coding region. $\mathrm{K}=\mathrm{G}$ or $\mathrm{T}, \mathrm{R}=\mathrm{A}$ or $\mathrm{G}, \mathrm{S}=\mathrm{C}$ or $\mathrm{G}, \mathrm{Y}=\mathrm{C}$ or $\mathrm{T}$.

40\%. These findings suggest there have been repeated exchanges between the Pokey A and PokeyB elements and the IGS sequences of the rDNA units.

In addition to $\mathrm{A}$ and $\mathrm{C}$ repeats, there are three other short repeated sequences in the $5^{\prime}$ and $3^{\prime}$ NCR of Pokey elements from the Daphnia genome that are shared with the mPok elements (Figure 2 and Additional file 6). Some of these repeats may correspond to repeat sequences previously found in other piggyBac elements, such as the one diagrammed in Additional file 6 from Trichoplusia ni [18].

\section{Target site preferences for Pokey and mPok}

Previous characterization of Pokey target sites found no preference aside from the requisite TTAA observed for all piggyBac elements [21-23]. However, in contrast to piggyBac elements, about $10 \%$ of the Pokey and $\mathrm{mPok}$ insertions, all oriented in the $5^{\prime}$ to $3^{\prime}$ direction, were found with target site duplications other than TTAA (Table 2). These other insertion sites were either TTAT or ATAA suggesting the only essential nucleotides are the middle $\mathrm{T}$ and $\mathrm{A}$. Insertion of piggyBac elements into non-TTAA sites has also been observed in transposition assays in bat $(7.2 \%,[24])$ and human cell lines $(2.4 \%$, [25]). In both cases, the alternate sites contained the middle $\mathrm{T}$ and $\mathrm{A}$.

Unlike insertions outside the rDNA locus, all Pokey and $\mathrm{mPok}$ elements but one insert at a single site in $28 \mathrm{~S}$ genes. The exception was an $\mathrm{mPok}$ sequence inserted into ITS2 near the sequence that gave rise to repeat $C$ in
Pokey elements. The specificity of Pokey elements for the $28 \mathrm{~S}$ gene site, despite the presence of over 30 TTAA sites in the rDNA unit, suggests that a larger recognition sequence could be involved in Pokey insertions. We therefore re-evaluated the flanking sequences of Pokey and $\mathrm{mPok}$ insertions outside of $28 \mathrm{~S}$ genes. About $23 \%$ of $\mathrm{mPok}$ and $7 \%$ of Pokey copies are inserted into the TTAA flanking another Pokey or mPok insertion (that is, they are organized as tandem repeats), and were excluded from the analysis. Visualization of preferred bases at specific sites revealed a weak preference for several bases immediately surrounding Pokey insertions (Figure 7). Significant sequence preferences included a $C$ one base and a T four bases upstream (5') of the TTAA, and a total of eight preferred bases downstream (3') of the TTAA: a $\mathrm{G}$ at position 4, an A at position 7, the sequence AAATG at positions 11 to 15 and a $\mathrm{T}$ at position 18 . Remarkably, each of these preferred bases match the Pokey target site in the $28 \mathrm{~S}$ gene.

\section{Discussion}

\section{Pokey diversity in the Daphnia genome}

Analysis of over 160 Pokey and Pokey-like sequences from the $D$. pulex genome revealed four well-supported clusters. Two clusters of larger elements with an average size of 5,100 bp were designated PokeyA and PokeyB. The clusters have diverged in sequence by about $40 \%$, have different ITR structures and include members that possess an intact transposase ORF. The two other clusters are MITEs, designated $\mathrm{mPok} 1$ and $\mathrm{mPok} 2$, because 


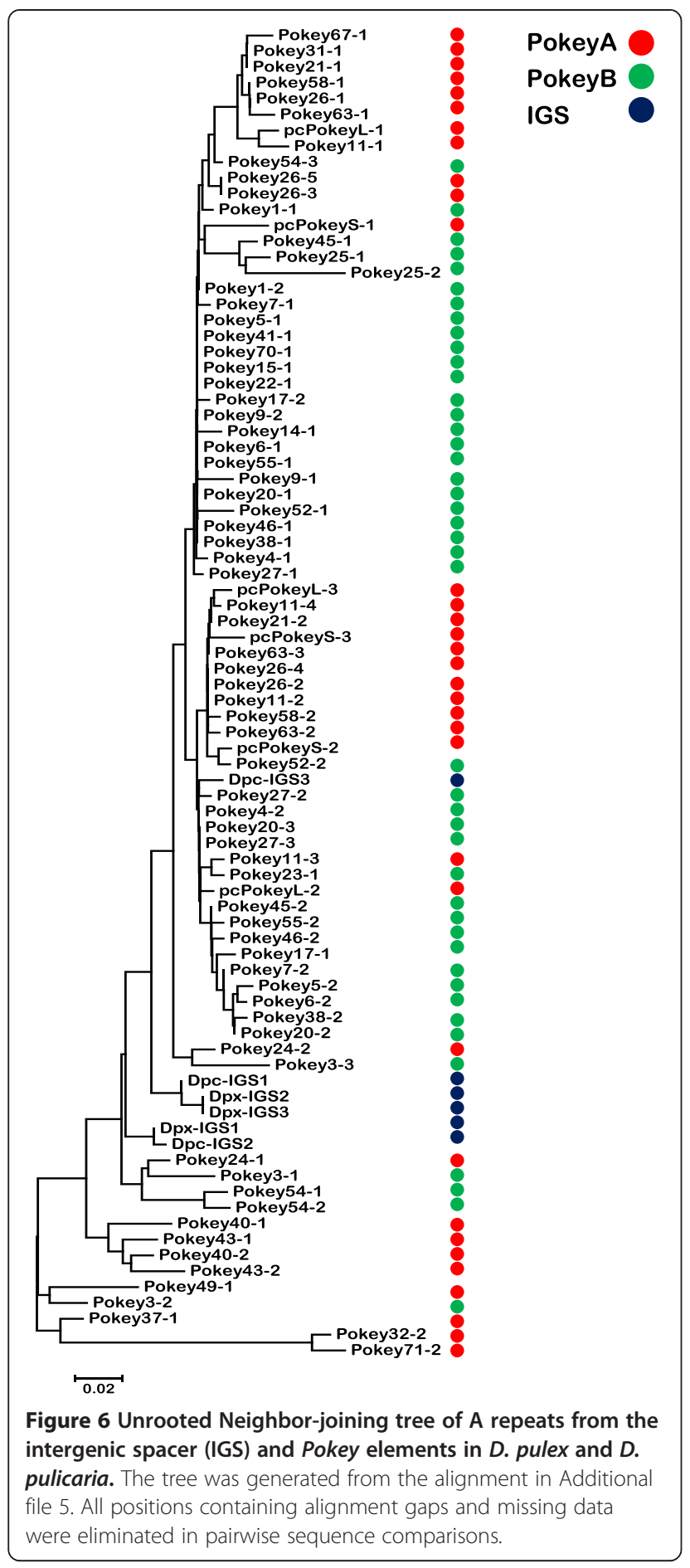

each mPok element contains an ITR and other noncoding sequences corresponding to one of the fulllength Pokey elements. Annotated PokeyB and mPok2 elements outnumber PokeyA and mPok1 elements by over 4:1.

Available evidence suggests that both PokeyA and PokeyB occur in D. obtusa [7] and thus the two lineages have likely persisted across multiple speciation events.
Table 2 Target site duplications flanking Pokey elements in the Daphnia genome sequence

\begin{tabular}{cc}
\hline Target site duplications & \% of insertions \\
\hline TTAA & 88.13 \\
TTAT & 5.93 \\
ATAA & 5.09 \\
CTAA & 0.85 \\
\hline
\end{tabular}

Vertical diversification of TEs within the same genome can be driven by drift, selection, or more likely a combination of the two. Two models have been proposed. Lampe and colleagues [26] observed a loss of interaction between the ITRs and transposases of Tc1/mariner elements from different subfamilies with sequence divergence greater than $16 \%$. They postulated that silencing mechanisms based on sequence similarity might create intragenomic selection that favors divergence of the transposase and ITR sequences of related TEs to escape silencing. A second possibility is that the presence of numerous non-autonomous elements drives the divergence of transposase and ITR sequences because the nonautonomous copies titrate the transposase from autonomous copies and decrease their fitness [27]. In that case, intragenomic selection might favor divergent elements whose transposases can only recognize their own ITRs.

The ability of PokeyA and PokeyB elements to crossmobilize could be investigated using yeast excision, yeast one-hybrid and/or electrophoretic mobility shift assays to determine the strength of interaction between the transposases and ITRs of each group. Although the differences in sequence between the two ITR structures appear minor (Figure 1), Casteret and colleagues [28] demonstrated that a small number of single nucleotide changes to the ITR of the drosophilid DNA transposon Mos1 produced significant changes in transposition rate.

The mPok elements appear to be of an atypically large size (approximately $750 \mathrm{bp}$ ) compared to other MITEs, which can be as small as around $130 \mathrm{bp}$ [29]. However, MITEs that are even larger than mPok have now been discovered in phylogenetically diverse eukaryotes (reviewed in [30]) suggesting that large MITEs are more common than once thought. One mechanism to explain the origin of large MITEs is progressive internal deletion of autonomous DNA TEs and subsequent selection for increasing transposition rate among the resultant elements over time [31]. Thus, the larger size of mPok elements could be a consequence of their recent evolution. While this could be true for the mPok1 elements, which show little sequence diversity, the occurrence of highly divergent $\mathrm{mPok} 2 \mathrm{~b}$ copies is not consistent with a recent origin (Figure 4). Indeed, Deprá and colleagues [32] suggested that the Mar MITEs in Drosophila willistoni, which are similar in size to the mPok elements, may 


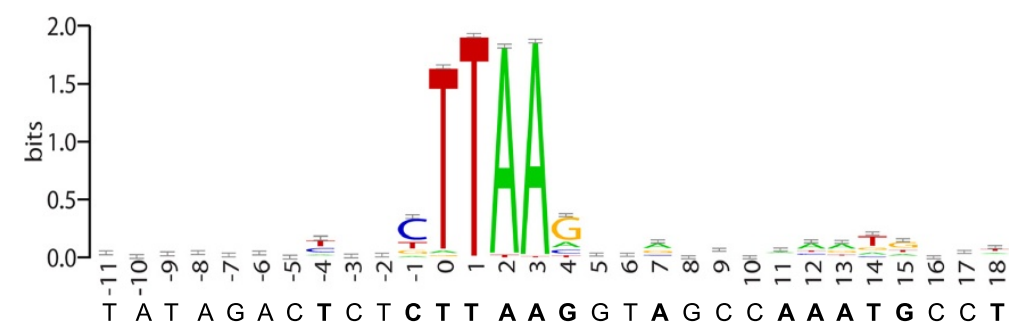

$28 \mathrm{~S}$ gene target sequence

Figure 7 Sequences flanking the TTAA target site of Pokey and Pokey-derived miniature inverted repeat transposable elements

(mPok). WebLogo was used to analyze $26 \mathrm{bp}$ upstream and $26 \mathrm{bp}$ downstream of the TTAA site. The analysis is based on 130 unique sequences upstream and 162 sequences downstream of the target site from the Daphnia genome. The corresponding sequence in the Daphnia 285 gene is shown below the graph. Positions in the $28 \mathrm{~S}$ gene that match preferred positions in genomic insertion sites are in bold type.

have originated prior to the diversification of the willistoni subgroup 5.7 MYA, suggesting that large size does not necessarily indicate recent origin.

\section{Repeated sequences in Pokey}

An unusual aspect of the PokeyA and B lineages in Daphnia is the presence of sequences derived from NCRs of the rDNA unit. This includes an approximately 200 bp sequence from a non-repetitive region of the IGS (A repeats) and an approximately 50 bp sequence from ITS2 (C repeats) (Figures 1 and 5). Pokey elements contain from 2 to 5 copies of these rDNA sequences within their 5' NCR (Figure 5). The highly recombinogenic nature of these repeats within the Pokey elements was first suggested by their differential spacing in pcPokeyS and pcPokeyL [3] and is strongly supported by this analysis in which particular combinations of $\mathrm{A}$ and $\mathrm{C}$ repeats are unique to only one or a few Pokey elements.

The acquisition of DNA to the $5^{\prime}$ NCR of Pokey does not appear to be limited to rDNA. For example, Pokey62 contains a unique, approximately $3,600 \mathrm{bp}$ sequence of which approximately $1,100 \mathrm{bp}$ is derived from sequence on a non-rDNA scaffold in the Daphnia genome. Thus, Pokey elements often acquire sequences from their host's genome. Langer and colleagues [33] proposed that Ds elements could acquire host sequence if the transposase slides after binding but before cutting, or if cryptic ITRlike sequences exist downstream of an element. However, the acquisition of sequences well within the $5^{\prime}$ NCR of the Pokey elements argues against such a simple explanation (Figure 5).

What is the significance of the $A$ and $C$ repeats? It is possible that they have no function and that their origin was chance recombination events that had no fitness impact on Pokey. However, the finding that all but one copy of Pokey from both lineages contain these repeats suggests that they do play some role in Pokey activity. Possible functions of these sequences include transcription enhancers, transcription terminators to prevent the formation of aberrant rRNA read-through transcripts, or binding sequences to recruit epigenetic modifiers [34-36]. We suggest a transcription role for these repeats to be most likely as mPok elements, which do not need to be transcribed to be mobilized by a Pokey transposase, do not have the rDNA repeats.

The most remarkable property of the A repeats is that the same sequence was retained in both the PokeyA and B lineages. Not only do the A repeats correspond to the highest level of sequence conservation between the two lineages, but the A repeats within the two Pokey lineages are as well conserved as IGS sequences undergoing concerted evolution within the rDNA unit (Figure 6). This high level of sequence identity suggests that recombination between the Pokey repeats and the rDNA repeats occurs on a regular basis, thus strengthening the argument that Pokey elements have become highly specialized for their insertion into the rDNA locus.

\section{Target site selection and the rDNA niche}

While it is not possible to assemble the sequences of individual Pokey elements inserted in rDNA, it should be noted that the consensus Pokey sequence from rDNA is similar to an assembled non-rDNA copy that could putatively encode a functional transposase (Figure 3). Given the rapid turnover of rDNA units, Pokey elements within the locus should be among the newest insertions, while those outside of rDNA are a combination of new and old insertions.

Pokey is the only DNA-mediated TE that is known to evolve insertion specificity for the rDNA unit. Remarkably, the Pokey insertion site is in the same region of the $28 \mathrm{~S}$ gene that is also the target site for a number of nonLTR retrotransposons (reviewed in [2]). Two of these elements, R2 and R5, which insert within a few base pairs of the Pokey site, encode related endonucleases that have an active site similar to class IIS restriction enzymes $[37,38]$. The R2 endonuclease has been shown to have exceptional specificity for the 30 to 40 nucleotides 
surrounding its insertion site $[39,40]$. Two other nonLTR retrotransposons, R1 and R4, insert 75 and $28 \mathrm{bp}$, respectively, downstream of the Pokey site. These elements encode an endonuclease with similarity to the apurinic endonuclease involved in DNA repair [41]. The endonuclease encoded by $\mathrm{R} 1$ has also been shown to have sequence specificity for the insertion site $[41,42]$. In all four cases, most copies of the element are inserted in rDNA with most copies outside rDNA inserted into sites with sequence similarity to the $28 \mathrm{~S}$ gene target site $[13,43]$.

The transposase of Pokey elements represents a third protein that has evolved specificity for this region of the $28 \mathrm{~S}$ gene. Some of the best-studied examples of integrases that have evolved insertion specificity involve the LTR retrotransposons of yeast [44-47]. In these cases, the integrases have evolved protein-protein specificity for association with specific transcription factors or chromatin structural components rather than actual DNA sequence specificity. Such protein-chromatin interactions could also be involved in the insertion specificity of Pokey elements, but we are not aware of any specific chromatin components that are bound to the central region of $28 \mathrm{~S}$ genes. Alternatively, the A repeat associated with Pokey elements may contain a recognition site for a nucleolar protein that helps guide into the nucleolus Pokey elements that have been excised and are ready for insertion.

It seems a remarkable coincidence that three different lineages of TEs have evolved specificity for the same small region of the rDNA unit. The $28 \mathrm{~S}$ target region is highly conserved, but there are many regions of the $18 \mathrm{~S}$ and $28 \mathrm{~S}$ genes that are conserved across eukaryotes. We suggest either the DNA in this region is highly exposed and thus accessible to the TE machinery, a yet unknown chromatin component can be utilized by the TE in its evolution of specificity, or this is one of only a few areas of the rDNA where a TE can insert without being quickly eliminated by recombination or selected against by the synthesis of disrupted rRNA.

Based on the concordance between phylogenies of rDNA Pokey elements and their hosts, Penton and Crease [7] concluded that Pokey has undergone stable, vertical inheritance in the rDNA of species in the subgenus Daphnia since its origin. Thus, unlike most Class II TEs, Pokey elements appear to have evaded complete silencing by the host for millions of years. The unique breeding system of Daphnia, involving extended periods of apomictic reproduction, and the complete loss of sexuality in some lineages may have created strong selection pressure on ancestral Pokey elements to avoid causing deleterious mutations in their host, while still maintaining a transposition rate high enough to survive. The theory describing the interaction between TEs and asexual or partially asexual hosts predicts three possible outcomes: (1) active elements are lost, (2) the host goes extinct due to TE-induced mutation, or (3) the elements become domesticated and the threat is neutralized [48]. However, Pokey's invasion of rDNA suggests a fourth outcome, the long-term persistence of active elements.

Zhou and colleagues [5] have argued that rDNA is an ideal TE niche, because it is difficult for the host to completely silence elements that have inserted into genes that must be expressed. In addition, TEs inserted in the locus are continually removed by recombination events so old copies that could interfere with the elements are eliminated. Finally, each insertion has a predictable, small effect on the fitness of the host. This effect is small because all organisms contain more than enough rDNA for the production of rRNA, and those rDNA units with insertions are usually not transcribed [49]. R2 and R1 elements, which are abundant in the rDNA of arthropods including crustaceans, have not been found in Daphnia. Perhaps Pokey elements are even better adapted for this niche in that they can be lost from the rDNA locus, but copies located outside the rDNA can on occasion be active and re-establish insertions in the locus. Indeed, individual $D$. pulex that lack PokeyA in rDNA have been observed, but no individuals have been observed that completely lack Pokey elements [11,19,50-52].

\section{Conclusions}

In spite of what would appear to be a seemingly inhospitable location for a DNA transposon, Pokey has evolved specificity for a site in the $28 \mathrm{~S}$ genes of Daphnia. Analysis of both the annotated $D$. pulex genome and the raw trace files revealed that rDNA units display extremely low levels of sequence variation consistent with the high rates of recombination previously observed for this locus. Indeed, Pokey has diversified into two lineages of autonomous elements, PokeyA and PokeyB, which appear to have persisted across multiple speciation events. While members of the $\mathrm{B}$ lineage are located in the rDNA of the population in Oregon that was selected for genomic sequencing [15], members of the A lineage are in the rDNA of $D$. pulicaria and $D$. pulex populations outside Oregon [3,7,52]. Both Pokey lineages have given rise to two parallel lineages of MITES, mPok1 and $m P o k 2$, which appear to be deletion derivatives of the full-length elements.

Part of the specificity of Pokey elements can be attributed to the sequence specify of the transposase itself, as the target site of non-rDNA copies bears weak sequence similarity to the $28 \mathrm{~S}$ rRNA insertion site. However, both Pokey lineages possess repeat sequences derived from rDNA that vary in arrangement and copy number. These repeats may play a role in the expression of Pokey elements from the rDNA locus, and/or a role in insertion 
specificity. Whatever their function, the Pokey repeats are evolving in concert with each other and with the rDNA unit itself suggesting ongoing sequence exchange. It remains unknown whether Pokey elements in or out of the rDNA locus are most active, and what fraction of new insertions occur in rDNA. While more insertions are found outside rDNA, this could simply reflect the fact that non-rDNA insertions are more stable over time. Overall, our results suggest a complex interaction between Pokey and its host, and highlight the need to concentrate not only on host traits but also on traits of individual families when trying to understand the current dynamics and past evolutionary history of TEs.

\section{Methods}

\section{Search for and assembly of rDNA and Pokey elements}

The original sequencing reads of the genome sequencing project from the cladoceran crustacean Daphnia pulex were accessed by basic local alignment search tool (BLAST) [53,54] in the Trace Archives at GenBank [55]. In addition, BLAST searches were conducted of the assembled scaffolds at wFleaBase [56].

The search for Pokey elements in $28 \mathrm{~S}$ genes was conducted in the same manner as searches for other 28S-specific TEs in rDNA [57]. Briefly, a BLAST search was conducted using the downstream region flanking the Pokey insertion site as the query. Reads identified in this search were examined upstream of the query region for sequences that were not $28 \mathrm{~S}$ and thus putative TEs. Once the consensus of the TE end was acquired, iterative BLAST searches were conducted using the end of each newly acquired TE extension until the $5^{\prime}$ junction of the element with the $28 \mathrm{~S}$ gene was reached. In order to identify copies present outside $28 \mathrm{~S}$ genes, the ends of the TE consensus sequences were used as BLAST queries and the flanking sequences examined. Sequences of the putative transposase gene were analyzed using the PSORTII server [58] to identify features of the amino acid sequence.

\section{Cluster analysis of Pokey elements}

Pokey elements were aligned using a combination of the CLUSTAL, MUSCLE and MAFFT multiple sequence alignment programs available from the EMBL-EBI website [59]. Alignments were manually adjusted in the program BioEdit [60]. Only sequences with less than $5 \%$ ambiguous bases across the aligned region and containing an ITR at both ends were used in cluster analyses. Measurements of pairwise sequence divergence were calculated using the Kimura 2-parameter method [61] in MEGA4 [62]. NJ trees [63] were also constructed in MEGA4. Bootstrap analysis was performed on 1000 pseudo-replicates for each tree [64]. The alignment of full-length elements excluded the variable repeat region between the 5' ITR and the transposase gene. In addition, a dataset including the last approximately $1,600 \mathrm{bp}$ of the 3' end of rDNA Pokey elements from species in the subgenus Daphnia [7] was aligned with the Pokey elements from the Daphnia genome sequence and used to generate an NJ tree.

\section{Sequence variation in rDNA and Pokey elements}

Sequence variation present in the rDNA transcription units and in a $500 \mathrm{bp}$ region of the IGS was evaluated in the same manner as described by Stage and Eickbush [13]. Briefly, 525 bp overlapping regions of each consensus were used as BLAST queries in the trace archives. Approximately 250 reads were collected from each BLAST search and evaluated for sequence changes present in at least eight sequence reads. In order to screen out sequencing errors, sites containing sequence differences were further evaluated using the trace quality scores available through the trace archives at GenBank [55].

A total of 26 base pairs on each side of the Pokey insertion site of both Pokey and mPok elements, all oriented in the $5^{\prime}$ to $3^{\prime}$ direction, were compared to determine if a preferred base is present at each position. A graphical representation of sequence conservation was made using WebLogo [65]. Only the 4 bp upstream and 15 bp downstream of the insertion contain preferred bases.

\section{Analysis of repeat sequences in Pokey}

Identification of repeat sequences within Pokey, and comparisons between Pokey and rDNA were performed using Pustell DNA matrix in MacVector 10.0 (MacVector Inc., Cary, NC, USA). Default parameters were used with $80 \%$ sequence identity in a $16 \mathrm{bp}$ window.

\section{Additional files}

Additional file 1: Consensus sequences of the rDNA unit, Pokey and mPok from the Daphnia genome sequence. The sequences are provided in Fasta format. The highly length-variable region at the $5^{\prime}$ end of Pokey elements has been omitted and is indicated by several Xs.

Additional file 2: List of Pokey elements extracted from the annotated scaffolds of the Daphnia genome sequence. The scaffold number (S), first nucleotide position (nt), length in bp (length) and lineage (PokeyA or B, mPok1 or 2) is provided for each sequence. NJ, Neighbor-joining tree.

Additional file 3: Unrooted Neighbor-joining tree of $1600 \mathrm{bp}$ sequences from the $3^{\prime}$ end of Pokey elements. Elements from the Daphnia genome sequence and cloned from the rDNA of other species in the subgenus Daphnia [7] are included. The latter are preceded by PC. All positions containing alignment gaps and missing data were eliminated in pairwise sequence comparisons. Bootstrap values greater than 70 are shown at the nodes in the tree.

Additional file 4: Partial alignment of transposase amino acid sequences from Pokey and piggyBac-superfamily elements. The three conserved catalytic aspartic acid (D) residues, the four cysteine (C) residues thought to compose the zinc-finger/Plant Homeo Domain (PHD) motif and the putative nuclear localization signal (NLS) are highlighted. The asparagine $(\mathrm{N})$ residue conserved in Pokey transposases is 
highlighted in grey. Other piggyBac elements have $D$ at this position. $\mathrm{pB}-\mathrm{Bmor}$, putative Bombyx mori piggyBac transposase; pB-Harm, piggyBac transposase from Helicoverpa armigera; $\mathrm{BB}$-Xtro, piggyBac transposase from Xenopus tropicalis; pB-like-Hsap, piggyBac transposase-derived protein from Homo sapiens.

Additional file 5: Alignment of A repeats from the IGS and Pokey elements in Daphnia pulex and Daphnia pulicaria. The sequences of 76 Pokey A repeats and the corresponding sequence from three ribosomal IGS from each of D. pulex and D. pulicaria [12] are provided in Fasta format. The order of the repeat within an element is given after the element name (for example, Pokey11-3 is copy 3 in element 11). The number of A repeats ranges from 2 to 5 per element.

Additional file 6: Repeated sequences in Pokey with similarity to piggyBac elements. The approximate location of repeat sequences in piggyBac that lack primary sequence identity with those in Pokey, but occur in similar locations, are indicated for both elements. The dashed line in Pokey presents the repetitive region described in Figure 5. The repetitive region, $5^{\prime} \mathrm{NCR}$ and transposase genes are not drawn to scale. $\mathrm{NCR}$, non-coding region; tpase, transposase gene.

\section{Abbreviations}

BLAST: Basic local alignment search tool; Bp: Base pair; D: Aspartate; IGS: Intergenic spacer; ITR: Inverted terminal repeat; LTR: Long terminal repeat; MITE: Miniature inverted repeat transposable element; mPok: Pokeyderived miniature inverted repeat transposable element; N: Asparagine; NCR: Non-coding region; NJ: Neighbor-joining; ORF: Open reading frame; rDNA: ribosomal DNA; rRNA: ribosomal RNA; RT-PCR: Reverse transcriptase polymerase chain reaction; TE: Transposable elements.

\section{Competing interests}

The authors declare that they have no competing interests.

\section{Authors' contributions}

THE and TJC conceived the project. TAE analyzed Pokey elements from the annotated genome sequence. DES analyzed rDNA and Pokey elements from the trace files. All authors wrote the manuscript and approved the final version.

\section{Acknowledgements}

Funding for this work was provided by a grant from the NIH (GM42790) to THE and an NSERC Discovery Grant to TJC.

\section{Author details}

'Department of Integrative Biology, University of Guelph, Guelph, ON N1G 2W1, Canada. ${ }^{2}$ Department of Biology, University of Rochester, Rochester, NY 14627, USA. ${ }^{3}$ Department of Biology, Butler County Community College, Butler, PA 16002, USA.

\section{Received: 18 July 2013 Accepted: 29 August 2013}

Published: 23 September 2013

\section{References}

1. Levin HL, Moran JV: Dynamic interactions between transposable elements and their hosts. Nat Rev Genet 2011, 12:615-627.

2. Eickbush TH, Eickbush DG: Finely orchestrated movements: evolution of the ribosomal RNA genes. Genetics 2007, 175:477-485.

3. Penton EH, Sullender BW, Crease TJ: Pokey, a new DNA transposon in Daphnia (Cladocera: Crustacea). J Mol Evol 2002, 55:664-673.

4. Zhang $X$, Eickbush MT, Eickbush TH: Role of recombination in the longterm retention of transposable elements in rRNA gene loci. Genetics 2008, 180:1617-1626.

5. Zhou J, Eickbush MT, Eickbush TH: A population genetic model for the maintenance of R2 retrotransposons in rRNA gene loci. PLoS Genet 2013, 9:e1003179.

6. Balu B, Shoue DA, Fraser MJ Jr, Adams JH: High-efficiency transformation of Plasmodium falciparum by the lepidopteran transposable element piggyBac. Proc Natl Acad Sci U S A 2005, 102:16391-16396.

7. Penton EH, Crease TJ: Evolution of the transposable element Pokey in the ribosomal DNA of species in the subgenus Daphnia (Crustacea: Cladocera). Mol Biol Evol 2004, 21:1727-1739.
8. Xu H-F, Xia Q-Y, Liu C, Cheng T-C, Zhao P, Duan J, Zha X-F, Liu S-P: Identification and characterization of piggyBac-like elements in the genome of domesticated silkworm, Bombyx mori. Mol Genet Genomics 2006, 276:31-40.

9. Jurka J: Repbase update: a database and an electronic journal of repetitive elements. Trends Genet 2000, 9:418-420.

10. Gladyshev EA, Meselson M, Arkhipova IR: Massive horizontal gene transfer in Bdelloid rotifers. Science 2008, 320:1210-1213.

11. Valizadeh P, Crease TJ: The association between breeding system and transposable element dynamics in Daphnia pulex. J Mol Evol 2008, 66:643-654.

12. Ambrose C, Crease T: Evolution of the nuclear ribosomal DNA intergenic spacer in four species of the Daphnia pulex complex. BMC Genet 2011, 12:13.

13. Stage DE, Eickbush $T H$ : Sequence variation within the rRNA gene loci of 12 Drosophila species. Genome Res 2007, 17:1888-1897.

14. Stage $D E$, Eickbush $T H$ : Maintenance of multiple lineages of $R 1$ and $R 2$ retrotransposable elements in the ribosomal RNA gene loci of Nasonia. Insect Mol Biol 2010, 19:37-48.

15. Colbourne JK, Pfrender ME, Gilbert D, Thomas WK, Tucker A, Oakley TH, Tokishita S, Aerts A, Arnold GJ, Basu MK, Bauer DJ, Cáceres CE, Carmel L, Casola C, Choi JH, Detter JC, Dong Q, Dusheyko S, Eads BD, Fröhlich T, Geiler-Samerotte KA, Gerlach D, Hatcher P, Jogdeo S, Krijgsveld J, Kriventseva EV, Kültz D, Laforsch C, Lindquist E, Lopez J, et al: The ecoresponsive genome of Daphnia pulex. Science 2011, 331:555-561.

16. Glass SK, Moszczynska A, Crease TJ: The effect of transposon Pokey insertions on sequence variation in the 28S rRNA gene of Daphnia pulex. Genome 2008, 51:988-1000.

17. McTaggart SJ, Dudycha JL, Omilian A, Crease TJ: Rates of recombination in the ribosomal DNA of apomictically propagated Daphnia obtusa lines. Genetics 2007, 175:311-320.

18. Li X, Lobo N, Bauser CA, Fraser MJ: The minimum internal and external sequence requirements for transposition of the eukaryotic transformation vector piggyBac. Mol Genet Genomics 2001, 266:190-198.

19. Vergilino R, Elliott TA, Desjardins ProulX P, Crease TJ, Dufresne F: Evolution of a transposon in Daphnia hybrid genomes. Mobile DNA 2013, 4:7.

20. Keith JH, Schaeper CA, Fraser TS, Fraser MJ: Mutational analysis of highly conserved aspartate residues essential to the catalytic core of the piggyBac transposase. BMC Mol Biol 2008, 9:73.

21. Wilson MH, Coates CJ, George AL: PiggyBac transposon-mediated gene transfer in human cells. Mol Ther 2007, 15:139-145.

22. Wang J, Dua Y, Wang S, Brown SJ, Park Y: Large diversity of the piggyBaclike elements in the genome of Tribolium castaneum. Insect Biochem Mol Biol 2008, 38:490-498.

23. Wang J, Miller ED, Simmons GS, Miller TA, Tabashnik BE, Park Y: piggyBaclike elements in the pink bollworm, Pectinophora gossypiella. Insect Mol Biol 2010, 19:177-184.

24. Mitra R, Fain-Thornton J, Craig NL: piggyBac can bypass DNA synthesis during cut and paste transposition. EMBO J 2008, 27:1097-1109.

25. Li X, Ewis H, Hice RH, Malani N, Parker N, Zhou L, Feschotte C, Bushman FD, Atkinson PW, Craig NL: A resurrected mammalian $h A T$ transposable element and a closely related insect element are highly active in human cell culture. Proc Natl Acad Sci U S A 2012, 109:22-27.

26. Lampe DJ, Walden KKO, Robertson HM: Loss of transposase-DNA interaction may underlie the divergence of mariner family transposable elements and the ability of more than one mariner to occupy the same genome. Mol Biol Evol 2001, 18:954-961.

27. Feschotte C, Pritham EJ: DNA transposons and the evolution of eukaryotic genomes. Annu Rev Genet 2007, 41:331-368.

28. Casteret S, Chbab N, Cambefort J, Auge-Gouillou C, Bigot Y, Rouleux-Bonnin F: Physical properties of DNA components affecting the transposition efficiency of the mariner Mos1 element. Mol Genet Gen 2009, 282:531-546.

29. Bureau TE, Wessler SR: Tourist: a large family of small inverted repeat elements frequently associated with maize genes. Plant Cell 1992, 4:1283-1294.

30. Fattash I, Rebecca Rooke R, Amy Wong A, Caleb Hui C, Luu T, Bhardwaj P, Yang G: Miniature inverted-repeat transposable elements: discovery, distribution, and activity. Genome 2013, 56:1-12.

31. Feschotte C, Zhang X, Wessler SR: Miniature inverted-repeat transposable elements and their relationship to established DNA transposons. In Edited by Craig NL, Craigie R, Gellert M, Lambowitz AM. Edited by Mobile DNA II. Washington DC: American Society for Microbiology Press; 2002:1147-1158 
32. Deprá M, Ludwig A, Valente VLS, Loreto ELS: Mar, a MITE family of hAT transposons in Drosophila. Mobile DNA 2012, 3:13.

33. Langer M, Sniderhan LF, Grossniklaus U, Ray A: Transposon excision from an atypical site: a mechanism of evolution of novel transposable elements. PLoS One 2007, 10:e965.

34. Kohorn BD, Rae PMM: A component of Drosophila RNA polymerase promoter lies within the rRNA transcription unit. Nature 1983, 304:179-181.

35. Mougey EB, Pape LK, Soliner-Webb B: Virtually the entire Xenopus laevis rDNA mulikilobase intergenic spacer serves to stimulate polymerase I transcription. J Biol Chem 1996, 271:27138-27145.

36. Ye J, Eickbush TH: Chromatin structure and transcription of the R1- and R2-inserted rRNA genes of Drosophila melanogaster. Mol Cell Biol 2006, 26:8781-8790.

37. Yang J, Malik HS, Eickbush TH: Identification of the endonuclease domain encoded by $\mathrm{R} 2$ and other site-specific, non-long terminal repeat retrotransposable elements. Proc Natl Acad Sci U S A 1999, 96:7847-7852.

38. Burke WD, Singh $D$, Eickbush $T H$ : R5 retrotransposons insert into a family of infrequently transcribed $28 \mathrm{~S}$ rRNA genes of planaria. Mol Biol Evol 2003, 20:1260-1270.

39. Xiong YE, Eickbush TH: Functional expression of a sequence-specific endonuclease encoded by the retrotransposon R2Bm. Cell 1988, 55:235-246.

40. Luan DD, Eickbush TH: Downstream $28 \mathrm{~S}$ gene sequences on the RNA template affect the choice of primer and the accuracy of initiation by the $\mathrm{R} 2$ reverse transcriptase. Mol Cell Biol 1996, 16:4726-4734.

41. Feng Q, Schumann G, Boeke JD: Retrotransposon R1Bm endonuclease cleaves the target sequence. Proc Natl Acad Sci U S A 1998, 95:2083-2088

42. Maita N, Aoyagi H, Osanai M, Shirakawa M, Fujiwara H: Characterization of the sequence specificity of the R1Bm endonuclease domain by structural and biochemical studies. Nucl Acids Res 2007, 35:3918-3927.

43. Xiong $Y$, Burke WD, Jakubczak JL, Eickbush TH: Ribosomal DNA insertion elements $\mathrm{R} 1 \mathrm{Bm}$ and $\mathrm{R} 2 \mathrm{Bm}$ can transpose in a sequence specific manner to locations outside the $28 \mathrm{~S}$ genes. Nucl Acids Res 1988, 16:10561-10573.

44. Kirchner J, Connolly CM, Sandmeyer SB: In vitro position-specific integration of a retrovirus-like element requires Pol III transcription factors. Science 1995, 267:1488-1491.

45. Bachman N, Eby Y, Boeke JD: Local definition of Ty1 target preference by long terminal repeats and clustered tRNA genes. Genome Res 2004, 14:1232-1247.

46. Dai J, Xie W, Brady TL, Gao J, Voytas DF: Phosphorylation regulates integration of the yeast Ty5 retrotransposon into heterochromatin. Mol Cell 2007, 27:289-299.

47. Guo Y, Levin HL: High-throughput sequencing of retrotransposon integration provides a saturated profile of target activity in Schizosaccharomyces pombe. Genome Res 2010, 20:239-248.

48. Nuzhdin SV, Petrov DA: Transposable elements in clonal lineages: lethal hangover from sex. Biol J Linn Soc 2003, 79:33-41.

49. Eickbush DG, Ye J, Zhang $X$, Burke WD, Eickbush TH: Epigenetic regulation of retrotransposons within the nucleolus of Drosophila. Mol Cell Biol 2008, 28:6452-6461.

50. Sullender BW, Crease TJ: The behavior of a Daphnia pulex transposable element in cyclically and obligately parthenogenetic populations. J Mol Evol 2001, 53:63-69.

51. Schaack S, Pritham EJ, Wolf A, Lynch M: DNA transposon dynamics in populations of Daphnia pulex with and without sex. Proc R Soc B 2010, 277:2381-2387.

52. Eagle SHC, Crease TJ: Copy number variation of ribosomal DNA and Pokey transposons in natural populations of Daphnia. Mobile DNA 2012, 3:4.

53. Altschul S, Gish W, Miller W, Myers E, Lipman D: Basic local alignment search tool. J Mol Biol 1990, 215:403-410.

54. BLAST. [http://blast.ncbi.n/m.nih.gov/Blast.cgi].

55. GenBank trace files. [http://www.ncbi.nlm.nih.gov/Traces/trace.cgi].

56. WFleaBase. [http://wfleabase.org].

57. Stage DE, Eickbush TH: Origin of nascent lineages and the mechanisms used to prime second-strand synthesis in the R1 and R2 retrotransposons of Drosophila. Genome Biol 2009, 10:R49.

58. PSORT II. [http://psort.hgc.jp/form2.html].

59. EMBL-EB/ alignment too/s. [http://www.ebi.ac.uk/Tools/sequence.html]

60. BioEdit. [http://www.mbio.ncsu.edu/BioEdit/page2.html].

61. Kimura M: A simple method for estimating evolutionary rate of base substitutions through comparative studies of nucleotide sequences. J Mol Evol 1980, 16:111-120.
62. Tamura K, Dudley J, Nei M, Kumar S: MEGA4: Molecular Evolutionary Genetics Analysis (MEGA) software version 4.0. Mol Biol Evol 2007, 24:1596-1599.

63. Saitou N, Nei M: The neighbor-joining method: a new method for reconstructing phylogenetic trees. Mol Biol Evol 1987, 4:406-425.

64. Felsenstein J: Confidence limits on phylogenies: an approach using the bootstrap. Evolution 1985, 39:783-791.

65. WebLogo. [http://weblogo.threeplusone.com]

doi:10.1186/1759-8753-4-20

Cite this article as: Elliott et al.: In and out of the rRNA genes: characterization of Pokey elements in the sequenced Daphnia genome. Mobile DNA 2013 4:20.

\section{Submit your next manuscript to BioMed Central and take full advantage of:}

- Convenient online submission

- Thorough peer review

- No space constraints or color figure charges

- Immediate publication on acceptance

- Inclusion in PubMed, CAS, Scopus and Google Scholar

- Research which is freely available for redistribution 